\title{
Artificial Intelligence based Composition for E-Government Services
}

\author{
Amina ADADI ${ }^{1}$. Mohammed BERRADA ${ }^{1}$. Nabil EL AKKAD ${ }^{1}$ \\ ${ }^{1}$ Computer and Interdisciplinary Physics Laboratory (LIPI), ENS Fez \\ Sidi Mohammed Ben Abdellah University \\ Fez, Morocco \\ amina.adadi@gmail.com
}

\begin{abstract}
Given the complex nature of the public sector with several distributed governmental institutions and manifold semantic differences of interpretation, the achievement of interoperability and integration is a key challenge for a comprehensive and effective Electronic Government (e-Government). Promising technologies that could be used to tackle this issue are: the powerful concept of ontology and the advanced Artificial Intelligence (AI) systems. Ontologies contribute to a common understanding of heterogeneous resources, while AI techniques make process integration dynamic and automated. However, up until now the use of AI along with ontologies has been fairly limited in e-Government. There is still, then, untapped potential in this field which worth to be exploited. In this paper, we present a dynamic approach for semantically integrating e-Government Web services based on AI techniques. The overall objective of our approach is to improve the citizen centric e-Government vision by providing a conceptual framework for automatically discovering, composing and optimizing eGovernment services. Within the proposed approach, special emphasis is put on personalization aspects and evaluation criteria for e-Government platform.
\end{abstract}

Keywords: Semantic Web Service Composition; e-Government; Ontology; Multi-agent systems; AI Planning; Machine Learning; Reinforcement Learning.

\section{Introduction}

Current trend in e-Government applications calls for joined-up services that shaped around and responding to the needs of the citizen, and not merely arranged for the public institutions' convenience. Setting up such a seamless e-Government services requires making the shift from isolated silos in public administration to one integrated government that encapsulates the size and the complexity of the administrative body and which provides interoperable and transparent services to citizen through a single point of access in the socalled one-stop government [1][2].

Technology is a key enabler of the envisioned one-stop government as it can bring real solutions to integration, interoperability, coordination and security issues. Indeed, several studies have been undertaken all over the world by different research communities in the field of e-Government services composition and integration [3][4][5]. Most of the recent eGovernment projects support the one stop shop e-services vision and use semantic technologies to deal with interoperability issues. But, to the best of our knowledge, no one of such projects has explored the potential of using distributed planning algorithms along with ontologies based on a multi agent architecture, to address the issues of semantic interoperability and service integration of e-Government.

Toward dynamic semantic Web service composition is the subject of our researches, as it has been argued in [6] our goal is to automate the whole composition process including discovery, composition, execution and interoperation of Web services. Our proposal stems from the following basic underlying idea: The synergy between four basic technologies 
namely; (i) Semantic Web [7], (ii) Multi-Agent System [8], (iii) Autonomic Computing paradigm [9] and (iv) Artificial Intelligent Planning (AIP) techniques [10], can lead to the development of a comprehensive platform that supports personalized, integrated and interoperable Web services. E-Government has some specific features as opposed to traditional e-business scenarios, the e-Government domain is a large, heterogeneous, dynamic and shared information space with various semantic differences of interpretation. Thus, eGovernment seems to be an obvious and promising application field for our semantic Web service composition approach. And since e-Government services require a high degree of interoperability and distribution due to the numerous entities they imply, this is where our semantic Web service composition approach can produce the best results to solve a significant number of e-Government issues. Therefore, the combination of these two areas is very much natural and beneficial.

The present paper is an extension of our previous work [6][11][12] that propose a redesigned version of our semantic Web service composition approach for e-government use with a focus on the personalization aspects.

The remainder of the paper is organized as follows: in Section 2 presens the layered architecture of the proposed platform. Section 3 details our proposed machine-learning model for enabling personalized services. Section 4 proposes a qualitative evaluation of the proposed approach. Section 5 gives our concluding remarks.

\section{The proposed architecture}

Fig. 1 shows the high-level overview of the proposed approach architecture. It is organized in a layered architecture that traces the service lifecycle from its request until it delivery. As depicted in Fig. 1 the platform consists of four layers, namely i) Legacy system layer, ii) Ontology layer, iii) Composition layer and iv) Communication layer.

- Legacy system layer: contains the legacy applications available in each of the public administrations (PA) involved in the system.

- Ontology layer: Ontologies [13], as powerful knowledge representation formalism for semantic modelling and annotation, were chosen as a basic mechanism to capture semantic content of e-Government Web services in a manner that can be automatically processed by a machine. Our system integrates: (a) e-Government domain Ontology that contains the domain's types, concepts and relations among them (a full implementation of our eGovernment domain Ontology can be found in [11]) . (b) e-Government Service Ontology plays the role of a UDDI registry with extended functionalities allowing the storing of a rich semantic description of Web services involved in PA services. (c) QoS Ontology specifies shared knowledge and vocabularies about the quality of service proprieties (QoS) and their relationship with respect to semantic services. (d) User profile Ontology incorporates concepts and properties used to model the user preferences and extra information, the latter plays a major role in the personalized process of the delivered services.

- Composition layer: Composition layer is the core component of the proposed architecture that provides the required functionalities such as discovery, composition and execution of services. It is composed of a set of intelligent agents capable of interacting and cooperating in order to co-build automatically a composition plan. The composition process is depicted under four phases namely: a) analysis, b) planning, c) evaluation and d) execution. For the sake of completeness the phases are just outlined here an extensive technical description of each phase can be found in [6]:

- Analysis: starting from an abstract specification of the user request, the aim of the analysis phase is to define semantically the requested service.

- Planning: during this phase a composition plan is generated in two steps namely, discovery and plan construction, the first step aims to find all candidate services that 
can potentially lead to satisfying the desired functionalities. The search is based on a matchmaking algorithm [6] using the service ontology repository and the domain ontology repository. In the second step, the Planner agent coordinates a set of Manager Agents that act on behalf of discovered semantic Web services. They work cooperatively using AIP techniques [6] to co-build an abstract composition plan.

- Evaluation: given a composition plan, an important challenge for the system is to find the "best" composite service execution plan with the respect of user specifications. The evaluation phase deals with this issue. It uses QoS ontology to rank the alternative concrete composite services through the use of quality of service techniques.

- Execution: finally, in the execution phase, the system invokes the selected service instances according to the execution plan and delivers the result back to the user.

- Communication layer: is the user interface that manages the interaction between the user and the system. This layer ensures a front office integration by proposing a single and unique access point to the e-Government services that integrates and merges any other access channel to these services.

The role of the proposed system is to lead the citizen from his/her problem to the appropriate service(s) by encapsulating the complexity of public institutions. Indeed, with an architecture based essentially on intelligent agents and ontologies, the middleware element formed by ontology and composition layers provides mechanisms to coordinate existent services in order to provide a new personalized service. Starting from an informal, incomplete and ambiguous request, intelligent agents explore e-Government services repository, where PA services are published in a semantic manner, to find the right combination of services that satisfies the user requirements, the use of AIP techniques makes this process dynamic and automatic. Then agents take in charge the execution of the composition process in a changing heterogonous and distributed environment based on an autonomic computing architecture and QoS techniques. As a result, the system provides a value added services that are more convenient for users' needs and perceptions and increase efficiency in terms of costs and productivity by reusing autonomous PA services while keeping their internal processes and legacy systems intact.

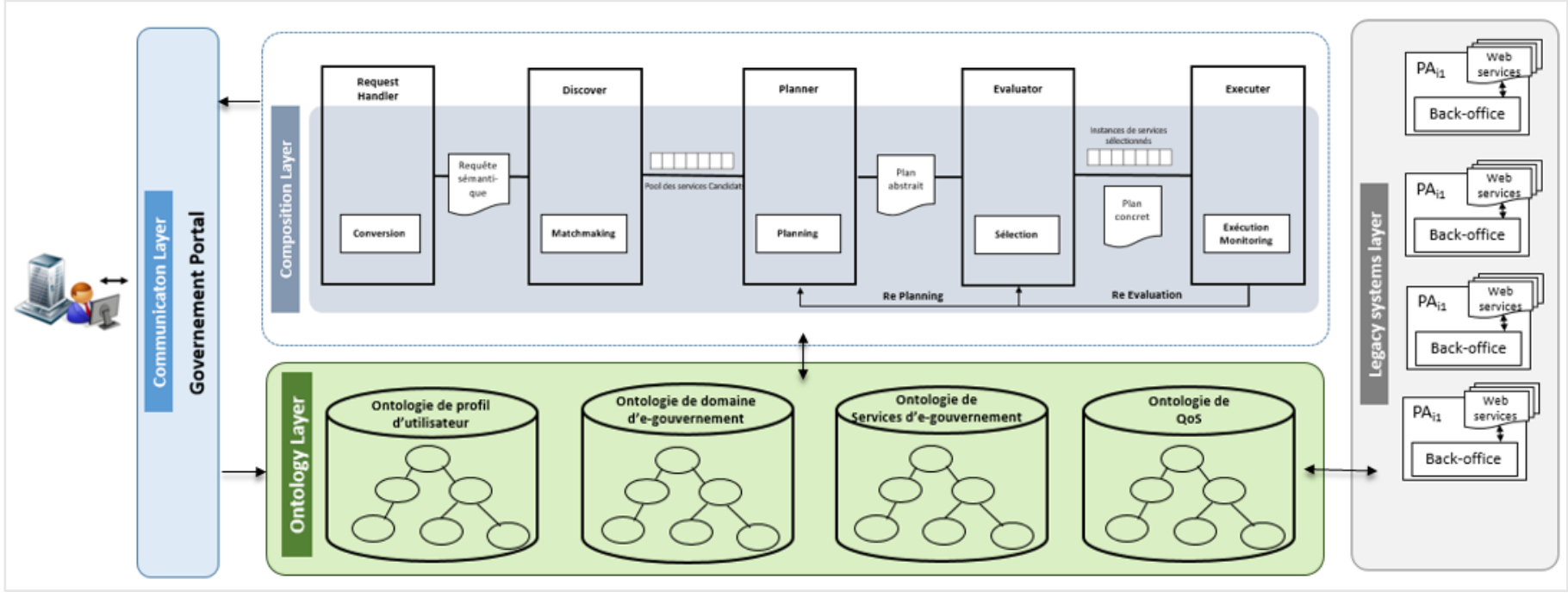

Fig. 1.The high level architecture of the proposed approach. 


\section{Recommendation layer: machine learning based personalization}

The proposed approach, as presented, focuses on interoperability and integration that represented long-standing challenges of e-government. In the era of the fourth industrial revolution, citizen collaboration is also seen as a key enabler for an efficient electronic government, thus more attention should be given to the personalized aspects of government services delivery.

This section aims at studying the use of machine learning algorithms for supporting personalized e-government services. To this purpose, we propose to combine our composition approach with a machine learning technique called: Reinforcement Learning (RL) [14]. RL is a process where an agent learns through experience by exploring the environment based on reward or the feedback. Typically, agents are not told which action to take, as in most forms of machine learning, but instead they must discover themselves which actions yield the most reward by trial and error. Thus, in this type of machine learning, the focus is shifted from pattern recognition to experience-driven sequential decision-making, which is suited for our problem that do not define a particular goal to be reached but a reward (defined by citizens) that has to be maximized.

Therefore, inspired by the successful use of RL in a range of fields including education, finance, manufacturing, and e-commerce. We advocate herein the promising potential application of RL in e-government to solve personalization related issues. Conceptually, a recommender system in form of a layer called "recommendation layer" will expand the capabilities of the actual composition approach to facilitate adaptivity by making "recommendations" to the system based on citizen feedback. As result, similarly to e-business where recommendation systems are used to help client locate information or products that they would like to make offers. Recommendation layer will allow citizens to benefit from the same experience in the e-government settings.

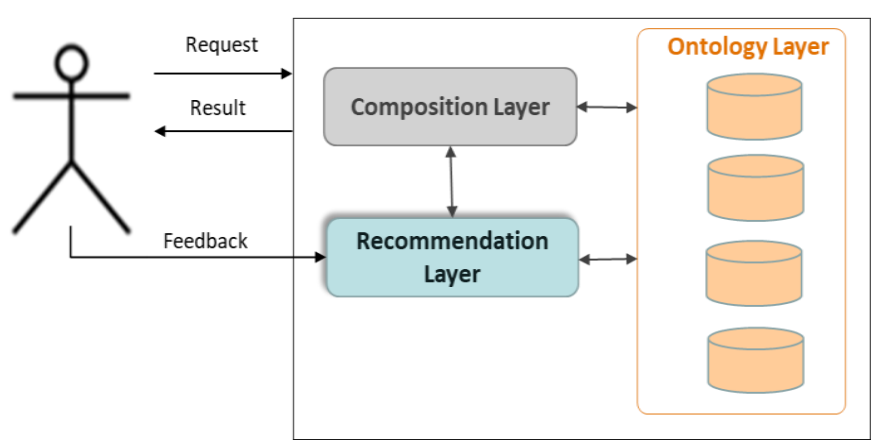

Fig. 2.The target combined approach with "Recommandation layer".

Fig.2 shows the main components and interaction processes of the target combined approach. The composition layer (that encapsulates the actual composition approach) and the recommendation layer are two distinct modules that interact with each other in order to generate personalized service that i) are formally correct with respect to user requests and ii) approximate implicit user requirements over time based on user ratings. This proposal is a starting point of a potential future work that needs to be deeply explored and developed in order to prove its relevance. 


\section{Evaluation and discussion}

Recognizing that e-Government should be transformative and become more citizen rather than government - focused in delivering public services, investing in the development of an e-government interoperability and integration framework is fundamental and promising, especially for developing countries. Indeed, in the Moroccan context, interoperability concepts and strategies are crucial agenda of the national e-Government development to achieve integrated, safe and efficient utilization of public services [15]. However, at a technical level, very few academic research works and studies have been focused on these issues. Thus, our work can be seen as an initiative that promotes an Artificial Intelligence based conceptual framework in response to interoperability and integration challenges of e-Government.

From our point of view, the seamless flow of data from one public administration to another can be achieved through ontologies. To support this idea, we developed, WebGov [11], an ontology for e-Government public services. WebGov fulfils the key requirements for a public services ontology to be useful [16]. Firstly, it covers multiple aspects of services, including administrative responsibility, involved documents, legislation and meta-data etc... Secondly, since it is designed and implemented based on METHONLOGY [17] method, the proposed ontology is fully extensible in the sense that it facilitates incremental ontology development, by incorporating basic concepts and relationships at an initial phase and then defining new ones, or creating instances as needed. The semantically rich network of interrelated concepts formulating by WebGovis mainly used in supporting the e-Government service composition tasks.

Furthermore, we proposed a conceptual framework populated by a toolkit of composition techniques that organize the e-Government integration process in a lifecycle. The use of Artificial Intelligence Planning algorithms and Multi-agent systems makes the proposed planning model dynamic and automated. While machine learning in form of RL has been identified as a key technology to meet the challenge of personalization aspects.

As a result, the overall approach represents an end-to-end conceptual framework that implements the following citizen/government relationship schema (Fig.3):

(i) $\mathrm{C} 2 \mathrm{G}$ and $\mathrm{G} 2 \mathrm{C}$ interactions that mark respectively the beginning and the end of the administrative process, they illustrate a front-office integration.

(ii) G2G interaction that encapsulates the mechanism of integrating (composing) the different public administration services in order to accomplish the administrative procedure requested by the citizen. This interaction illustrates a back-office integration.

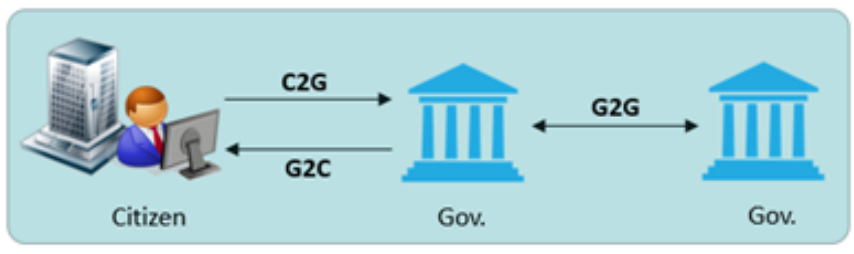

Fig. 3.The implemented Citizen/government relationship schema.

Evaluation plays a vital role in an approach validation process. At this stage of our work, we believe that a qualitative evaluation (QE) is the best tool that can lead us to reliable conclusions and can help us to identify the potential areas of improvement. Thus, in order to validate our approach, we conduct qualitative criteria based evaluation.

After having theorized extensively about evaluating Web service composition approaches [18][19][20][21] [21][23][24][25][26][27][28], We have noted that several evaluation criteria exist in the literature, however none of these evaluations take into account the e-Government specific requirements. The e-Government environment is highly heterogeneous in nature and manages a sensitive relationship (between government end citizens). Therefore, in order to perform a services composition in this domain, a composition approach must fulfils some 
particular criteria such as service relevance and security, citizens and business satisfaction and preservation of public trust, in addition of other standard requirements such as flexibility, reliability, accessibility, availability, processes automation, lower cost, short delivery time etc...Despite their diversity, by collecting Web service composition technical requirements spread across the literature and by analyzing their relationship, we have identified five core aspects of requirements: i) Expressiveness ii) Interoperability, iii) Transparency and iv) Adaptivity. These criteria are used to check how far the proposed approach supports a successful e-Government service composition process.

\section{- Expressiveness}

A granular expressiveness in service request and management is one of the important aspects that has to be taking in account.

The presented approach relies on a rich expressiveness of semantic descriptions of Web services (OWL-S), hence it supports functional and non-functional parameters such as Quality of Service (QoS). The approach also considers preference parameters to help the user in describing their requests at a granular way.

\section{- Interoperability}

As interoperability is the primary goal of most e-Government frameworks, ensuring interoperability needs to be a key focus item.

As explained before, our approach is driven by ontologies as a key instrument to ensure interoperability. In the same vein, dealing with service composition in heterogeneous and distributed systems like public administrations, is also an important and challenging issue. To face this challenge our approach is based on a multi-agent system architecture that allows a decentralized e-Government services integration.

\section{- Transparency}

A composition approach for e-Government services must be user (citizen and business) centric and hide the complexity of the service production processes, hence it has to optimize the human intervention (automation).

In the proposed approach, the citizen starts with a simple user interface where information from domain ontology and user profile ontology can be automatically loaded. Based on the planning model technique, the composition process is performed in an autonomic way and return back the result to the user who is shielded from the complexities of the background composition process. The composition process is also performed transparently from the service provider (public administration) without requiring intervention from their part, hence their internal systems remain intact during the composition cycles.

The utilization of proposed system is then completely transparent from the citizen and the public administration.

\section{- Adaptivity}

Adaptivity aspect has recently been attracting interest in research communities and involves the ability of a service (atomic or composite) to adapt itself. Adaptation is the process of modifying service-based applications in order to satisfy new requirements and to fit new situations dictated by the environment on the basis of predefined adaptation strategies. Adaptation goes one step further from dynamic criteria, in the sense that the former also deals with changes in the requirements set by the requester (citizen in our case) which the latter cannot handle. Moreover, adaptive approaches deliver personalized services which increases user satisfaction.

In our approach self-healing is a key aspect that takes in charge handling faults and recovering from problems reported during execution which increases the adaptivity of the system.

Moreover, by adding the recommendation layer, the approach will support user's feedback which can potentially help in delivering more personalized services. It will also involve learning from previous composition experiences, which implies an optimization of the composition process. 
As summarized in the table below, our approach is characterized by supporting QoS parameters, ontologies, self-healing and automated dynamic generation of composition schema based on the AI planning. However, the learning capacity is yet to be improved in order to improve the personalization aspect. Which reinforces, confirms, and motivates our proposal regarding the integration of the "Recommendation layer".

Table 1. Qualitative evaluation of the proposed approach.

\begin{tabular}{|l|l|}
\hline \hline Aspects & Level of fulfilment \\
\hline Expressiveness & + \\
\hline Interoperability & + \\
\hline transparency & + \\
\hline Adaptivity & $+/-$ \\
\hline \hline+ high & $+/$ - medium \\
\hline
\end{tabular}

\section{Conclusion}

In this paper, we studied how our approach for semantic Web services composition based on Artificial Intelligence techniques can be effectively applied to deliver citizen centric eGovernment services. Then we focused how to enable personalization based on machine learning.Finally, we evaluated qualitatively our proposal.

Our future work includes the completion of the proof-of-concept prototype but before, we plan to pursue this work by implementing applicative e-Government scenarios to extensively evaluate our approach's effectiveness. The use of machine learning is another extension way to be explored towards a more intelligent e-Government.

\section{References}

[1] Chatzidimitriou, M. Koumpis, A.: Marketing One-stop e-Government Solutions: the European OneStopGov Project, IAENG International Journal of Computer Science, Vol. 35, No. 1, (2008).

[2] United Nations.: Taking a whole of-government approach, E-Government Survey 2012, Chapter 3, (2012)

[3] Stojanovic, L. G. Kavadias, G.Apostolou, D. Probst, F. Hinkelmann, .: Ontology-enabled eGovServiceConfiguration, Delivrable Ontology Project - IST Project 507237,http://www.ontogov.com

[4] eGOIA - Electronic Government Innovation and Access, http://www.egoia.sp.gov.br/

[5] E.C 6th Framework Programme.: Electronic and Secure Municipal Administration for EuropeanCitizens - eMayor, IST-2004-507217, (2004), www.emayor.org

[6] Adadi, A.Berrada, M. Chenouni, D.: A phased two stages conceptual framework for Web service composition, Journal of Engineering and Applied Sciences (JEAS), 13 (7), (2018).

[7] Yu, W.Wen,G. Chen,G. Cao, J.: Distributed Cooperative Control of Multi-agent Systems, John Wiley \& Sons, (2016), 350 pp, DOI: 10.1002/9781119246213

[8] Laufer, C.: Semantic web guideline, government of the state of sãopaulo, Web Technologies Study Center, (2015)

[9] Abeywickrama, D.B. Ovaska, E.: A survey of autonomic computing methods in digital service ecosystems, Service Oriented Computing and Applications, Vol.11, Issue 1, pp: 1-31, (2017)

[10] M.Ghallab, M..Nau, D. Traverso, P. : Automated Planning and Acting, Cambridge University Press, (2016)

[11] Adadi, A.Berrada, M. Chenouni, D. Bounabat, B: Ontology based composition of e-Government services using AI Planning, the 10th International Conference on InelligentSystems : Theories and Applications (SITA'2015), (2015).

[12] Adadi, A.Berrada, M. Chenouni, D.:A Multi-Agent Planning Architecture for Semantic Web Service Composition, International Review on Computers and Software (IRECOS), (2014)

[13] L. Zhu,L. Tao,W. Min, X. Gao ,W. : Theoretical Characteristics of Ontology Learning Algorithm in Multi-dividing Setting, IAENG International Journal of Computer Science, Vol. 43 Issue 2, p184-191, (2016). 
[14] Arulkumaran, K. Deisenroth, M.Brundage, M.Bharath, A,:Deep Reinforcement Learning: A Brief Survey,IEEE Signal Processing Magazine , 34 (6), (2017)

[15] Vrandecic, D.: Ontology Evaluation: thesis, Karlsruhe Institute of Technology (KIT),Germany, 2010.

[16] CHAKRI, S.:Maroc digital 2020: Stratégiesnationales pour le développement de l'économienumérique, 2016, https://www.itu.int/en/ITU-D/RegionalPresence/ArabStates/Documents/events/2016/DigitalTransformation/Pres/Strat\%C3\%A9gies\%20na tionales\%20pour\%201e\%20d\%C3\%A9veloppement\%20de\%201\%E2\%80\%99\%C3\%A9conomie\% 20num\%C3\%A9rique\%2C\%20samia\%20chakri.pdf

[17] Sawsaa,A. F. Lu,J. : Building Information Science Ontology (OIS) with Methontology and Protégé, Journal of Internet Technology and Secured Transactions (JITST), Vol.1, Issue 4, (2012)

[18] Dustdar, S. Schreiner,W. .: "A survey on web services composition", Int. Journal of Web and Grid Services, vol. 1, no.1, pp. 1-30, (2005).

[19] Mantovaneli Pessoa, R. .Silva, E. Sinderen, M.V. Quartel,D. Pires, L.F. :"Enterprise Interoperability with SOA: a Survey of Service Composition Approaches", in Workshop on Enterprise Interoperability, pp. 32-45, (2008).

[20] Rao. J.. Su, X.: "A Survey of Automated Web Service Composition Methods", in Proceedings of the First International Workshop on Semantic Web Services Web Process Composition, (2004).

[21] Khadka, R. Sapkota,B. .: "An Evaluation of Dynamic Web Service Composition Approaches ”, In: 4th International Workshop on Architectures, Concepts and Technologies for Service Oriented Computing, (2010).

[22] Tabatabaei,S.G.H. Wan Mohd,K. Suhaimi,I. : "A Comparative Evaluation of State-of-the-Art Approaches for Web Service Composition", in Proceedings of Third International Conference on Software Engineering Advances, pp. 488-493, (2008).

[23] Beek, M. Bucchiarone,A. Gnesi,S. : "Formal Methods for Service Composition", Annals of Mathematics, Computing \&Teleinformatics, vol. 1, no. 5, pp. 1-10, (2007).

[24] Benatallah., B. :Service Composition: Concepts, Techniques, Tools and Trends in Service-Oriented Software System Engineering-Challenges and Practices, Idea Group Publishing, USA , pp. 48-66, (2005).

[25] Zuniga,J.C. Pérez-Alcazar, J. Digiampietri,L. : "Implementation Issues for Automatic Composition of Web Services", Workshops on Database and Expert Systems Applications, pp. 201-205, (2010).

[26] Sheng,Q.Z. Qiao, . X. Vasilakos, A.V. Szabo, C. Bourne,S. Xu,X.: Web services composition: A decade's overview, Information Sciences, Vol. 280, pp: 218-238, (2014)

[27] lagares, A. Florian, D. Boualem,B. : Web Service Composition: A Survey of Techniques and Tools, ACM Computing Surveys (CSUR), Vol. 48, Issue , (2016)

[28] AlSedrani,A. Touir,A. Web service composition in dynamic environment: a comparative study, The Fourth International Conference on Database and Data Mining, pp. 75-84, (2016), DOI: $10.5121 /$ csit.2016.60508 